\title{
Early recombinant factor VIla therapy in acute intracerebral hemorrhage: Promising approach
}

\author{
Sudhir Kumar, H. R. Badrinath \\ Department of Neurological Sciences, Apollo Hospitals, Hyderabad - 33, India
}

Intracerebral hemorrhage $(\mathrm{ICH})$ is the most devastating form of stroke with a high morbidity and mortality. $\mathrm{ICH}$ constitutes about $20-30 \%$ of all strokes, with the prevalence being higher in Asian population. Treatment of $\mathrm{ICH}$ is predominantly conservative, which includes control of blood pressure, use of anti-cerebral edema measures such as mannitol and mechanical ventilation. The benefit of early surgery in $\mathrm{ICH}$ is debatable. Initial hematoma volume and subsequent growth in its size are important predictors of a poor outcome in $\mathrm{ICH}$. This means that therapies aimed at preventing hematoma enlargement in the earliest possible window period could lead to a better outcome in $\mathrm{ICH}$. Recombinant factor VIla (rFVIla) is one such agent, which has been shown to prevent hematoma expansion and improve outcome in acute $\mathrm{ICH}$. The purpose of the current review is to focus on the evidence regarding the usefulness of rFVIla in acute $\mathrm{ICH}$.

Key words: Recombinant factor VIla, Acute intracerebral hemorrhage.

\section{Introduction}

Currently, intracerebral hemorrhage (ICH) is the most devastating and least treatment-amenable form of stroke. ICH is a relatively common neurological emergency and carries a high morbidity and mortality. Treatment is largely supportive and surgery is either non-beneficial or benefits only a small percentage. However, a new hope has been generated with the introduction and use of activated recombinant factor VII ( $\mathrm{rFVHa}$ ) in patients with ICH. The current article focuses on the rationale, mechanism of action, usefulness and safety profile of this agent.

\section{Epidemiology of ICH}

In comparison to ischemic stroke and subarachnoid hemorrhage, ICH patients suffer higher mortality and are left with more severe deficits. ${ }^{[1]}$ Over one-third of patients with ICH die within one month after onset of symptoms, and only $20 \%$ regain functional independence. ${ }^{[2]}$ ICH constitutes $15 \%$ of all strokes in the United States and Europe and $20 \%$ to $30 \%$ in Asian populations. ${ }^{[1]}$ Advancing age, hypertension and cerebral amyloid angiopathy are the most important risk factors for ICH. Contrary to major advances made in the acute treatment of other types of stroke, till date there is no effective treatment for ICH. ${ }^{[3]}$

\section{Pathophysiology of ICH}

Today it is recognized that the volume of the hematoma is an important determinant of mortality and functional outcome after $\mathrm{ICH}^{[4,5]}$ and early hematoma growth is an important cause of neurological deterioration. An increase in volume of more than 33 percent is detectable on repeated computed tomography (CT) in 38 percent of patients initially scanned within three hours after onset; in two thirds of cases with growth in volume, this increase is evident within one hour. ${ }^{[6]}$ Early hematoma growth occurs in the absence of coagulopathy and appears to result from continued bleeding or rebleeding at multiple sites within the first few hours after onset. ${ }^{[7]}$ Several predisposing factors for the enlargement of spontaneous ICH have been identified. Kazui et al, in a study on 186 patients with ICH, concluded that the following factors independently predisposed to hematoma enlargement: history of brain infarction; liver disease; fasting plasma glucose $>140 \mathrm{mg} /$ $\mathrm{dL}$ and systolic blood pressure on admission $>$ or $=200 \mathrm{~mm}$ Hg; glycosylated hemoglobin A1c $>$ or $=5.1 \%$ and systolic blood pressure on admission $>$ or $=200 \mathrm{~mm} \mathrm{Hg.}{ }^{[8]}$ In another study, a particularly high likelihood of hematoma enlargement was observed in patients who were admitted shortly after onset, who were heavy drinkers; who had an irregularly shaped hematoma, whose consciousness was disturbed, and who had a low level of fibrinogen. ${ }^{[9]}$ 3D-CT angiography performed within 12 hours has been utilized to document extravasation suggestive of continued hemorrhage, and these findings correlate well with 
hematoma enlargement as detected on noncontrast CT at 24 hours. ${ }^{[10]}$ The presence of contrast extravasation into the hematoma after CT angiography is associated with a significantly increased mortality $(63.5 \%$ versus $16.4 \%$ in patients without extravasation). ${ }^{[11]}$

\section{Rationale for the use of rFVIla in ICH}

Given the evidence that ongoing bleeding can occur for several hours after ICH onset, it is not without rationale that ultra-early hemostatic intervention could be helpful in limiting hematoma size with consequent improved clinical outcome. As is the case with all stroke treatments, hemostatic therapy must be given as soon as possible (preferably within 3-4 hours) after the onset of ICH to be effective. Although this narrow time-window for interventional therapy for all types of stroke is challenging, the feasibility of early hemostatic intervention in ICH is supported by the fact that ICH patients reach the emergency care facility relatively earlier than ischemic stroke patients. Early stoppage of hematoma progression reduces the frequency of neurological deterioration by preventing not only early worsening related to hematoma growth, but also by preventing late deterioration (>12 hours after onset) related to perihematomal edema and mass effect, which is most often a problem once a large hemorrhage has been established.

Ideally, an agent that is acceptable for early hemostasis in ICH should be the one that inhibits fibrinolysis and activates coagulation locally, allowing fast and effective hemostasis without the risk of systemic activation of coagulation cascade. Recombinant factor VIIa (rFVIIa; NovoSeven ${ }^{\circledR}$ ), for many years now, has been available for the management of bleeding in hemophilia patients with inhibitory antibodies to replacement Factor VIII/IX. Recently rFVIIa has also been approved for use in congenital FVII deficiency and Glanzmann's thrombasthenia in European Union. Furthermore, a large number of case series studies and anecdotal evidences, from patients with different bleeding conditions, have now shown that rFVIIa is actually a valuable general haemostatic agent. Earlier reluctance to use it as a general haemostatic agent resulted, in part, from a lack of understanding of its compartmentalized mode of action. However, as rFVIIa has been used successfully to treat various coagulopathies, and as we now have an increased understanding of its mode of action (MOA), there is a distinct possibility that it is a potential therapeutic adjunct for controlling hemorrhage in nonhemophiliac indications. ${ }^{[12]}$ This medical logic is supported by a growing body of evidence that suggests rFVIIa may have an important role as a valuable hemostatic in ICH, trauma, surgery; liver disease-associated bleeds, thrombocytopenia, and anticoagulant overdosage or toxicity. The spectrum of indications for rFVIIa albeit wide, our current endeavor is to focus on rFVIIa pharmacology and key clinical studies that describe the use of rFVIIa in ICH patients without preexisting coagulopathies.

\section{rFVIla - Mechanism of action}

rFVIIa has an amino acid sequence identical to that of plasmaderived rFVIIa and is produced from a baby hamster kidney cell line, cultured in bovine albumin. Being a biotechnology product, it is not derived from human or animal plasma, thus eliminating the risk of human blood transmitted diseases. $r \mathrm{FVII}$ is usually administered by slow IV bolus injection and it has a predictable, well-characterized pharmacokinetic profile (half-life of 2.7 hours).

The exact mode of action of rFVIIa remains to be established. Current data based on its effect in hemophilia patients suggest that it provides local thrombin generation by enhancing the TF/ FVIIa complex at the injury site, thereby augmenting the TFdependent pathway of hemostasis. The TF/FVIIa complex then initiates the coagulation cascade by activating factors X and IX. According to cell-based model of hemostasis, high-dose of rFVIIa enhances hemostasis in hemophiliacs by binding to activated platelets with low affinity, and activating FX independently of TF. The FXa produced in this way is sufficient to partially restore platelet-surface thrombin generation in hemophiliacs, leading to the subsequent production of fibrin (Figure 1). ${ }^{[12]}$ rFVIIa essentially works in the same way in ICH patients, augmenting the normal coagulation process to produce a faster and more pronounced thrombin burst. In ICH setting, rFVIIa therefore has two potential sites of action: one at the ruptured small arteries and arterioles, by binding to exposed TF to initiate the coagulation process; and the other on the surface of activated platelets, to produce thrombin independently of TF. These suggest that the effects of rFVII are localized to the site of vascular injury, without systemic activation of the coagulation process. ${ }^{[13]}$ Thrombotic potential is therefore reduced. Although the half-life of rFVIIa is only $2.6 \mathrm{~h}$, a sustained hemostatic effect may occur after a single dose because the clot that forms is denser than normal clot and more resistant to fibrinolysis. ${ }^{[14]}$

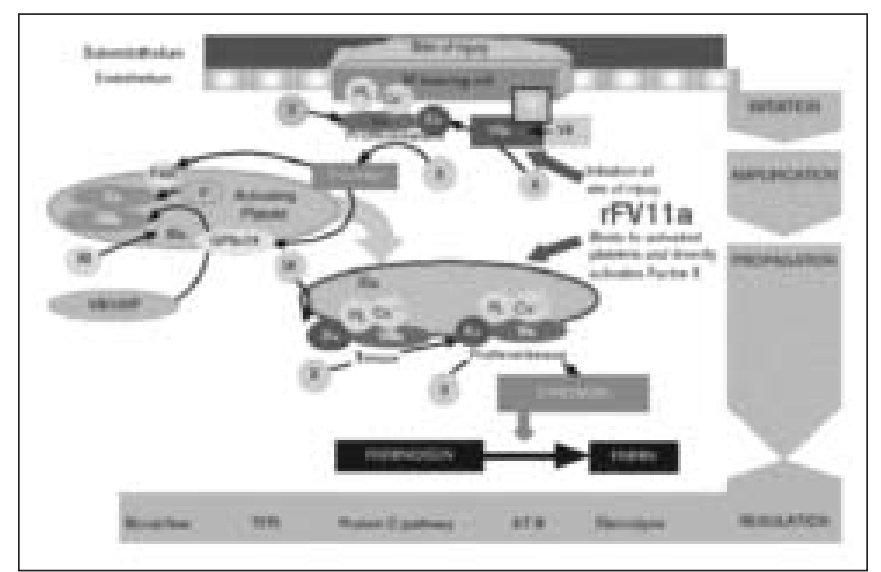

Figure 1: Schematic diagram depicting the mechanism of action of recombinant activated factor VII 


\section{Europe-AustralAsia rFVIla Pilot Study in ICH}

Although rFVIIa has a good safety profile in patients with hemophilia and other bleeding disorders, experience with this agent in older, noncoagulopathic patients with risk factors for cardiovascular disease was considered limited. Accordingly, the investigators' main objective in this study was to focus on treatment-related thrombo-embolic or coagulation-related adverse events. In this multi-centric, randomized, double-blind, placebocontrolled, dose-escalation trial by Mayer et al, ${ }^{[15]} 48$ subjects with ICH diagnosed within 3 hours of onset were treated with placebo $(n=12)$ or rFVIIa $(10,20,40,80,120$, or $160 \mathrm{meg} / \mathrm{kg} ; \mathrm{n}=6$ per group). Mean time from symptom onset to treatment was 181 minutes, and the mean "CT-to-needle" time was 69 minutes. In this pilot study, rFVIIa was generally well-tolerated, and only 6 possible treatment-related adverse events occurred, including 2 cases of DVT (one each in the placebo and $20 \mathrm{mcg} / \mathrm{kg}$ groups). Overall there were 12 serious adverse events, including one of the aforementioned cases of DVT, and a patient in the $20 \mathrm{meg} / \mathrm{kg}$ dose group in whom unstable angina developed 29 days after randomization, which was judged as unlikely to be related to treatment. The other 10 serious adverse events were nonthromboembolic in nature, consistent with the natural history of ICH, and deemed unlikely to be related to treatment. Thrombin has been hypothesized as edema-generating. ${ }^{[16]}$ To evaluate whether rFVIIa exacerbated perihematoma edema, edema-to-ICH volume ratios were compared between the dose groups; with respect to this, authors found no significant differences between placebo and various rFVIIa dosage arms. This study also found no evidence of subclinical activation of systemic coagulation, which is in accordance with findings in patients with hemophilia and in warfarin-treated normal volunteers. Finally this pilot study concluded that acute treatment of ICH patients with rFVIIa is feasible and safe across a wide range of rFVIIa doses. Another parallel US study of $40 \mathrm{ICH}$ patients that tested a lower range of doses has demonstrated a similar safety profile. ${ }^{[17]}$

\section{Combined safety data from Europe-Australasian and US phase Ila study}

Skolnick et al ${ }^{[18]}$ have documented combined safety data from the above mentioned two rFVIIa dose-escalation trials (EuropeAustralasia $^{[19]}$ and US ${ }^{[17]}$ Phase II studies). This randomized controlled safety trial compared placebo with 7 dose tiers of rFVIIa $(5,10,20,40,80,120$ and $160 \mathrm{meg} / \mathrm{kg})$. Patients had spontaneous ICH confirmed by CT sean within 3 hours of onset and were dosed within 1 hour of baseline CT. A total of 88 patients were studied; 68 received rFVIIa, 20 received placebo. Adverse events were evenly distributed among treatment groups. Despite the compromised status of this patient population, only 27 patients experienced 39 serious adverse events, 5 of which were thromboembolic events. Three thrombotic events occurred in rFVIIatreated patients (pulmonary embolism in patients in the 20 and $40 \mathrm{mcg} / \mathrm{kg}$ groups; myocardial infarction in a patient in the 80 mug/kg group), compared with 2 thrombotic events in placebotreated patients (deep vein thrombosis, cerebral infarction). No dose-related increase in the ratio of perihematomal edema to ICH volume was noted. Authors concluded that rFVIIa was safe within the investigated dose- range, subsequent to which larger study was planned to establish the efficacy and further safety of rFVIIa in ICH.

\section{rFVIla multi-centric ICH study}

In this placebo-controlled, three-dose tier, largest-to-date ICH medical intervention study, Mayer and colleagues ${ }^{[19]}$ randomly assigned 399 patients with ICH diagnosed by CT within three hours after onset to receive placebo (96 patients) or rFVIIa 40 $\mathrm{meg} / \mathrm{kg}$ (108 patients), $80 \mathrm{meg} / \mathrm{kg}$ (92 patients), or $160 \mathrm{mcg} / \mathrm{kg}$ (103 patients) within one hour after the baseline CT. The primary outcome measure was the percent change in the ICH volume at 24 hours. Clinical outcomes were assessed at 90 days. At 24-h CT, hematoma volume increased more in the placebo group than in the rFVIIa groups. The mean increase was 29 percent in the placebo group, as compared with 16 percent, 14 percent, and 11 percent in the groups given $40 \mathrm{mcg} / \mathrm{kg}, 80 \mathrm{mcg} / \mathrm{kg}$, and $160 \mathrm{mcg} /$ $\mathrm{kg}$ rFVIIa respectively ( $P=0.01 \mathrm{rFVII}$ a combined versus placebo). $69 \%$ of placebo-treated patients died or were severely disabled, as compared with 55 percent, 49 percent, and 54 percent of the patients who were given $40 \mathrm{mcg} / \mathrm{kg}, 80 \mathrm{mcg} / \mathrm{kg}$, and 160 $\mathrm{meg} / \mathrm{kg}$ of rFVIIa, respectively ( $P=0.004 \mathrm{rFVII}$ a combined versus placebo). Mortality at 90 days was 29 percent for patients who received placebo, as compared with 18 percent in the three rFVIIa groups combined $(P=0.02)$. Serious thrombo-embolic adverse events, mainly myocardial or cerebral infarction, occurred in 7 percent of rFVIIa- treated patients, as compared with 2 percent of those given placebo although not statistically significant $(P=0.12)$; rFVIIa apparently exhibited more predilection for arterial thrombotic events rather than for venous events. It is however worthwhile noting that there was no difference in the frequency of fatal or disabling thrombotic adverse events, that were possibly or probably related to treatment, between rFVIIa and the placebo groups, i.e. $2 \%$ in rFVIIa group and $2 \%$ in placebo group. Investigators concluded that rFVIIa given within $4 \mathrm{~h}$, albeit associated with a small risk of thrombo-embolic events, significantly reduces hematoma growth in a dose-dependent fashion, reduces mortality, and significantly improves global functional outcome at 90 days. It is worth mentioning here that the small risk of thrombotic adverse events with larger dose of rFVIIa has led to the choice of low and medium doses for the pivotal ongoing phase III trial.

\section{rFVIla - Tolerability profile}

Safety data from studies in other indications show that rFVIIa may not be associated with an increased risk of thrombo-embolic events. In support of this, there is a report by Roberts ${ }^{[20]}$ that reviewed clinical experience with rFVIIa between 1988 and May 
2001. During this time, more than 180,000 standard doses were administered to patients with hemophilia or other coagulation disorders, and even to healthy patients without any clinical evidence of a bleeding disorder. Only 17 patients experienced thrombotic events; of these, 11 patients had an arterial thrombotic event, and 6 demonstrated a venous thrombosis. A total of four patients died due to thrombo-embolic complications. Of the 17 patients, 3 demonstrated thrombotic symptoms either before or more than 10 days after rFVIIa administration, suggesting that the events were unlikely to be related to rFVIIa use. A further 6 patients were aged 70 years or older (range: 70-91 years), and suffered from one or more age-associated conditions, such as diabetes, atherosclerosis, and hypertension, all of which are risk factors for thrombosis in individuals with a normal coagulation system. Roberts $^{[20]}$ concluded that the reported incidence of rFVIIaassociated thrombo-embolic events was very low. Broderick ${ }^{[21]}$ observes that potential risks and adverse effects with rFVIIa treatment include ischemic stroke and other vascular events; since patients with ICH may also have a prior history of ischemic stroke, the risks of ischemic adverse events need to be monitored during rFVIIa ICH therapy. Though the studies mentioned above encourage the use of rFVIIa in the treatment of ICH and other indications, due caution is always advisable with respect to choosing proper patient population and optimal drug usage.

\section{Conclusions}

The hematoma volume is one of the important determinants of neurological and functional outcomes in patients with ICH and early hemorrhagic progression is an important cause of progressive neurological dysfunction. Hemostatic therapeutic intervention, given very early in the course of ICH, may thus improve clinical outcomes by arresting ongoing bleeding and limiting in turn the size of the hematoma. rFVIIa is approved to treat bleeding in hemophilia patients with inhibitors, congenital FVII deficiency and Glanzmann's thrombasthenia; it has also been reported to arrest bleeding in nonhemophiliac cases undergoing surgery or suffering trauma, ICH, liver diseases and anticoagulant toxicity. With respect to ICH, initial dose-escalation safety studies discussed above have demonstrated that doses of rFVIIa 5 to $160 \mu \mathrm{g} / \mathrm{kg}$ were relatively safe, though a low frequency of thrombotic adverse events was reported with higher doses. These encouraging results paved the way for subsequent larger randomized, multi-centric study, the objective of which was to corroborate the efficacy and safety of rFVIIa in ICH. Conclusions drawn from this largest-todate ICH medical intervention study imply that early rFVIIa hemostatic therapy, within 3-4 hours of symptom-onset, limits the growth of hemorrhage, reduces mortality, and improves functional outcomes with benefits that may outweigh the thromboembolic risk that exist with high and medium doses of factor VIIa.
More randomized trials are nevertheless needed, one Phase III study already underway, to further establish the therapeutic status of rFVIIa in ICH; this apart, future randomized trials should also focus on defining the optimal therapeutic window, appropriate patient population, optimal dosage and efficacy in the setting of thrombocytopenia or thrombo-prophylaxis induced ICH.

\section{References}

1. Saceo RL, Mayer SA. Epidemiology of intracerebral hemorrhage. In: Feldmann E, editor. Intracerebral Hemorrhage. Armonk, NY: Futura Publishing Co; 1994. p. $3-23$.

2. Counsell C, Boonyakarnkul S, Dennis M. Primary intracerebral hemorrhage in the Oxfordshire Community Stroke Project. 2. Prognosis. Cerebrovase Dis 1995;5:2634 .

3. Broderick JP, Adams HP Jr, Barsan W, Feinberg W, Feldmann E, Grotta J, et al. Guidelines for the management of spontaneous intracerebral hemorrhage:a statement for healtheare professionals from a special writing group of the Stroke Council, American Heart Association. Stroke 1999;30:905-15.

4. Broderick JP, Brott TG, Duldner JE, Tomsick T, Huster G. Volume of intracerebral hemorrhage:a powerful and easy-to-use predictor of 30-day mortality. Stroke $1993 ; 24: 987-93$.

5. Hemphill JC III, Bonovich DC, Besmertis L, Manley GT, Johnston SC. The ICH score:a simple, reliable grading scale for intracerebral hemorrhage. Stroke 2001;32:891-7

6. Brott T, Broderick J, Kothari R, Barsan W, Tomsick T, Sauerbeck L, et al. Early hemorrhage growth in patients with intracerebral hemorrhage. Stroke 1997;28:15 .

7. Mayer SA. Ultra-early hemostatic therapy for intracerebral hemorrhage. Stroke $2003 ; 34: 224-9$

8. Kazui S, Minematsu K, Yamamoto H, Sawada T, Yamaguchi T. Predisposing factors to enlargement of spontaneous intracerebral hematoma. Stroke 1997;28:2370-5.

9. Fujii Y, Takeuchi S, Sasaki O, Minakawa T, Tanaka R. Multivariate analysis of predictors of hematoma enlargement in spontaneous intracerebral hemorrhage. Stroke 1998;29:1160-6.

10. Murai Y, Takagi R, Ikeda Y, Yamamoto Y, Teramoto A. Three-dimensional computerized tomography angiography in patients with hyperacute intracerebral hemorrhage. J Neurosurg 1999;91:424-31.

11. Becker K.J, Baxter AB, Bybee HM, Tirschwell DL, Abouelsaad T, Cohen WA. Extravasation of radiographic contrast is an independent predictor of death in primary intracerebral hemorrhage. Stroke 1999;30:2025-32.

12. Hedner U. Recombinant factor Vlla (NovoSeven $\left.{ }^{\circledR}\right)$ as a haemostatic agent. Bloodline Reviews 2001;1:3-4

13. Hoffman M, Monroe DM, Roberts HR. Activated factor VII activates factors IX and $\mathrm{X}$ on the surface of activated platelets:thoughts on the mechanism of action of high-dose activated factor VII. Blood Coagul Fibrinol 1998;9:S61-5.

14. Erhardtsen E. Ongoing NovoSeven ${ }^{\circledR}$ trials. Intensive Care Med 2002;28:S24856.

15. Maver SA, Brun N, Broderick J, Davis S, Diringer MN, Skolnick BE, et al. Europe/ AustralAsia NovoSeven ICH Trial Investigators. Safety and feasibility of recombinant factor VIIa for acute intracerebral hemorrhage. Stroke 2005;36:74-9.

16. Lee KR, Colon GP, Betz AL, Keep RF, Kim S, Hoff JT. Edema from intracerebral hemorrhage:the role of thrombin. J Neurosurg 1996;84:91-6.

17. Mayer SA, Brun N, Davis S, Broderick J, Diringer MN, Steiner T. For the US NovoSeven ICH Trial Investigators. Safety and preliminary efficacy of recombinant coagulation factor VIIa in acute intracerebral hemorrhage: US Phase 2A Study. Stroke 2004;35:332

18. Skolnick BE, Brun NC, Kruse P, Seremetis SV. Safety and laboratory results for recombinant activated coagulation factor VII in patients with acute intracerebral hemorrhage. Blood 2003;102:307a.

19. Mayer SA, Brun NC, Begtrup K, Broderick J, Davis S, Diringer MN, et al. For the Recombinant Activated Factor VII Intracerebral Hemorrhage Trial Investigators. Recombinant activated factor VII for acute intracerebral hemorrhage. N Engl J Med 2005;352:777-85.

20. Roberts HR. Recombinant factor Vlla (NovoSeven $\left.{ }^{\circledR}\right)$ and the safety of treatment. Semin Hematol 2001;38:48-50

21. Broderick PJ. Advances in the treatment of hemorrhagic stroke: A possible new treatment. Cleveland Clin .J Med 2005;72:342-4.

Accepted on 14-11-2005 